\title{
Minimum Neighbor Distance Estimators of Intrinsic Dimension
}

\author{
Gabriele Lombardi, Alessandro Rozza, Claudio Ceruti, \\ Elena Casiraghi, and Paola Campadelli \\ Dipartimento di Scienze dell'Informazione, Università degli Studi di Milano, \\ Via Comelico 39-41, 20135 Milano, Italy \\ lombardi@dsi.unimi.it \\ http://homes.dsi.unimi.it/ campadel/LAIV/index.htm
}

\begin{abstract}
Most of the machine learning techniques suffer the "curse of dimensionality" effect when applied to high dimensional data. To face this limitation, a common preprocessing step consists in employing a dimensionality reduction technique. In literature, a great deal of research work has been devoted to the development of algorithms performing this task. Often, these techniques require as parameter the number of dimensions to be retained; to this aim, they need to estimate the "intrinsic dimensionality" of the given dataset, which refers to the minimum number of degrees of freedom needed to capture all the information carried by the data. Although many estimation techniques have been proposed, most of them fail in case of noisy data or when the intrinsic dimensionality is too high. In this paper we present a family of estimators based on the probability density function of the normalized nearest neighbor distance. We evaluate the proposed techniques on both synthetic and real datasets comparing their performances with those obtained by state of the art algorithms; the achieved results prove that the proposed methods are promising.
\end{abstract}

Keywords: Intrinsic dimensionality estimation, dimensionality reduction, manifold learning.

\section{Introduction}

Most of the machine learning techniques suffer the "curse of dimensionality" (Hughes effect) when applied to high dimensional data; for these reasons, many real life signals characterized by high dimensionality, such as images, genome sequences, or EEG data, cannot be successfully processed. To face this limitation, a first step of dimensionality reduction is needed to project the data onto lower dimensional spaces where they are tractable. This step could be profitable when the reduced data are projected along the most characterizing dimensions. Indeed, most real data can be fully characterized by few degrees of freedom, represented by low dimensional feature vectors; in this case the feature vectors can be viewed as points constrained to lie on a low dimensional manifold embedded

D. Gunopulos et al. (Eds.): ECML PKDD 2011, Part II, LNAI 6912, pp. 374-389, 2011.

(C) Springer-Verlag Berlin Heidelberg 2011 
in a higher dimensional space. The dimensionality of these manifolds is generally referred as intrinsic dimensionality (id). In other words, the id of a given dataset $\boldsymbol{X}_{N} \equiv\left\{\boldsymbol{x}_{i}\right\}_{i=1}^{N} \subset \Re^{D}$ is the minimum number of parameters needed to capture, and describe, all the information carried by the data. In more general terms, according to [11], $\boldsymbol{X}_{N}$ is said to have id equal to $d \in\{1 . . D\}$ if its elements lie entirely within a $d$-dimensional subspace of $\Re^{D}$.

Given a high dimensional dataset, the estimation of its id would be a fundamental step of any dimensionality reduction technique. Unfortunately, to our knowledge, existing techniques fail dealing with high id non-linear datasets, and the problem is still open.

In this work we present the following intrinsic dimension estimation methods: the "Minimum Neighbor Distance - Maximum Likelihood" estimator and its variants $\left(\mathrm{MiND}_{\mathrm{ML} *}\right)$, and the "Minimum Neighbor Distance - Kullback Leibler" estimator $\left(\mathrm{MiND}_{\mathrm{KL}}\right)$; moreover, we compare them with state of the art algorithms.

In Section 2 the related works are summarized; in Section 3 our theoretical results and our estimators are proposed; in Section 4 experimental settings and results are reported; in Section 5 conclusions and future works are presented.

\section{Related Works}

The most cited example of id estimator is the Principal Component Analysis (PCA) 14, which is a well known technique that is often used as the first step of many machine learning methods to reduce the data dimensionality. To this aim, PCA projects points on the directions of their maximum variance, which are estimated by computing the data covariance matrix and performing eigendecomposition. Exploiting PCA, the intrinsic dimension $d$ can be estimated by counting the number of normalized eigenvalues that are higher than a threshold parameter $\lambda$. In [10] the author achieves more accurate results by applying PCA in small subregions of the dataset to estimate their local id. The id of the whole dataset is then determined by combining all the local ids. The problem of using PCA relies in the difficulty of choosing a proper value for $\lambda$, which strongly affects the accuracy of the estimated id. Moreover, PCA is a linear technique and cannot successfully deal with non linear datasets.

Another well-known id estimator is the Packing Number technique [15. This method exploits the $r$-packing number $M(r)$ of the dataset $\boldsymbol{X}_{N} \subset \mathcal{S}$, where $\mathcal{S}$ is a metric space with distance metric $\delta(\cdot, \cdot)$. More precisely, $\boldsymbol{X}_{N}$ is said to be $r$-separated if $\forall \boldsymbol{x}, \boldsymbol{y} \in \boldsymbol{X}_{N}, \boldsymbol{x} \neq \boldsymbol{y} \Rightarrow \delta(\boldsymbol{x}, \boldsymbol{y}) \geq r$, and $M(r)$ is the maximum cardinality of an $r$-separated subset of $\boldsymbol{X}_{N}$. Given this definition, the authors demonstrate that the id of $\boldsymbol{X}_{N}$ can be found by approximating the limit:

$$
d=-\lim _{r \rightarrow 0} \frac{\log M(r)}{\log r} \quad \text { with } \quad \hat{d}=-\frac{\log \left(M\left(r_{2}\right)-M\left(r_{1}\right)\right)}{\log \left(r_{2}-r_{1}\right)}
$$

where $r_{2}>r_{1}$ are two radiuses to be set as parameters.

The previously described approaches often fail when managing non-linearly embedded manifolds or noisy data, and they become computationally too expensive when dealing with high dimensional datasets. 
To overcome these limitations, in [5] the authors propose an algorithm that exploits entropic graphs to estimate both the id of a manifold, and the intrinsic entropy of the manifold random samples. This technique is based on the observation that the length function of such graphs, that is the sum of arc weights on the minimal graph that spans all the points in the dataset, is strongly dependent on $d$. The authors test their method by adopting either the geodesic minimal spanning tree (GMST [4]), where the arc weights are the geodetic distances computed through the ISOMAP [23] algorithm, or the kNN-graph (kNNG [5]), where the arc weights are based on the Euclidean distances, thus requiring a lower computational cost.

The previously described algorithm, exploiting kNNG, can be inserted into the group of approaches, such as LLE [22, NN estimator [20], and TVF [19], that estimate the id by analyzing the relationships among nearest-neighbors within the data. Most of these methods consider hyperspheres with sufficiently small radius $r$ and centered on the points in the dataset, and they estimate some statistics by considering the neighboring points, included into the hypersphere; these statistics are expressed as functions of the intrinsic dimensionality of the manifold from which the points have been randomly drawn. One of these techniques is the Correlation Dimension (CD) estimator [12; it is based on the assumption that the volume of a $d$-dimensional set scales with its size $r$ as $r^{d}$, which implies that also the number of samples covered by a hypersphere with radius $r$ grows proportionally to $r^{d}$. Since the performance of the CD estimator is affected by the choice of the scale $r$, in [13] the authors suggest an estimator (which we will call Hein) based on the asymptotics of a smoothed version of the CD estimate. Another interesting approach is proposed in [8], where the author presented an algorithm to estimate the id of a manifold in a small neighborhood of a selected point, and they analyzed its finite-sample convergence properties. Another well known technique, based on the analysis of point neighborhoods, is the Maximum Likelihood Estimator (MLE) [17] that applies the principle of maximum likelihood to the distances between close neighbors, and derives the estimator by a Poisson process approximation. More precisely, calling $k$ the number of neighbors, $\boldsymbol{x}_{i}$ the $i$-th point, and $T_{k}\left(\boldsymbol{x}_{i}\right)$ the radius of the smallest sphere centered in $\boldsymbol{x}_{i}$ containing exactly $k$ neighbors, the local intrinsic dimension is estimated as:

$$
\hat{d}\left(\boldsymbol{x}_{i}\right)=\left(\frac{1}{k} \sum_{j=1}^{k} \log \frac{T_{k+1}\left(\boldsymbol{x}_{i}\right)}{T_{j}\left(\boldsymbol{x}_{i}\right)}\right)^{-1}
$$

To our knowledge, most of the neighborhood based estimators generally underestimate $d$ when its value is sufficiently high. To address this problem few techniques have been proposed, among which we recall the method described in [1]. In this work, Camastra et al. propose a correction of the estimated id based on the estimation of the error obtained on synthetically produced datasets of known dimensionality (hypercubes). 


\section{The Proposed Algorithms}

In this section we firstly present our theoretical results (see Section 3.1); according to them, we propose a maximum likelihood id estimator and its variants (see Section 3.2); moreover, we present an id estimation algorithm based on pdf comparison (see Section 3.3) which is more robust than state of the art methods with respect to high dimensional data. For the sake of clarity, in Appendix A the pseudocode of these algorithms is reported.

\subsection{Base Theoretical Results}

Consider a manifold $\mathcal{M} \equiv \Re^{d}$ embedded in a higher dimensional space $\Re^{D}$ through a locally isometric non-linear smooth map $\psi: \Re^{d} \rightarrow \Re^{D}$; to estimate the id of $\mathcal{M}$ we need to identify a "mathematical object", depending only on $d$, that can be estimated by means of points drawn from the embedded manifold.

To face this problem, we firstly consider the unit hypersphere $\boldsymbol{B}_{d}\left(\mathbf{0}_{d}, 1\right) \subset \Re^{d}$ centered in the origin and uniformly sampled; furthermore, we assume $\psi$ to be the identity map. Considering $k$ points $\left\{\boldsymbol{z}_{i}\right\}_{i=1}^{k}$ uniformly drawn from $\boldsymbol{B}_{d}\left(\mathbf{0}_{d}, 1\right)$, our aim is to find the pdf related to the minimum distance between the $k$ points and the hypersphere center $\mathbf{0}_{d}$.

Call $p(r)$ the pdf for the event $\left\|\boldsymbol{z}_{i}\right\|=r(r \in[0,1])$ where $\|\cdot\|$ is the $L_{2}$ norm operator, and denote with $P(\breve{r}<r)$ the probability for the event $\left\|\boldsymbol{z}_{i}\right\|<r$; being $\boldsymbol{z}_{i}$ uniformly drawn it is possible to evaluate these probabilities by means of hypersphere volume ratios. The volume of a $d$ dimensional hypersphere of radius $r$ is:

$$
V_{r}=r^{d} \frac{\pi^{d / 2}}{\Gamma\left(\frac{d}{2}+1\right)}=r^{d} V_{1}
$$

where $\Gamma(\cdot)$ is the Gamma function and $V_{1}$ is the volume of the unit $d$-dimensional hypersphere. The quantity $P(\breve{r}<r)$ is given by the volume ratio $\frac{V_{r}}{V_{1}}=r^{d}$; moreover, being $P(\breve{r}<r)$ the cumulative density function (cdf) related to the pdf $p(r)$, it is $p(r)=\partial \frac{V_{r}}{V_{1}} / \partial r=d r^{d-1}$.

The pdf $g(r ; d, k)$ related to the event $\min _{i \in\{1, \cdots, k\}}\left\|\boldsymbol{z}_{i}\right\|=r$ (i.e. the minimum distance between the points $\left\{\boldsymbol{z}_{i}\right\}_{i=1}^{k}$ and the hypersphere center equals to $r$ ) is proportional to the probability of drawing one point with distance $r$ multiplied by that of drawing $k-1$ points with distance $\breve{r}>r$, that is:

$$
\begin{aligned}
g(r ; d, k) & \propto \breve{g}(r ; d, k)=p(r)(1-P(\breve{r}<r))^{k-1}= \\
& =\frac{\partial \frac{V_{r}}{V_{1}}}{\partial r}\left(1-\frac{V_{r}}{V_{1}}\right)^{k-1}=\frac{1}{V_{1}} d r^{d-1}\left(1-r^{d}\right)^{k-1}
\end{aligned}
$$

Normalizing by $\int_{0}^{1} \breve{g}(r ; d, k) d r=\left(V_{1} k\right)^{-1}$ we finally get:

$$
g(r ; k, d)=\frac{\breve{g}(r ; d, k)}{\int_{0}^{1} \breve{g}(r ; d, k) d r}=k d r^{d-1}\left(1-r^{d}\right)^{k-1}
$$


Notice that Equation (1) holds only if we assume that the manifold is the unit radius hypersphere. Nevertheless, choosing a $d$-dimensional open ball $\boldsymbol{B}_{d}(\boldsymbol{c}, \epsilon)$ with center $\boldsymbol{c} \in \mathcal{M}$ and radius $\epsilon>0$, as long as $\psi$ is a non-linear smooth map that preserves distances in $\mathcal{B}_{d}$ and $\boldsymbol{z}$ is uniformly drawn from $\mathcal{B}_{d}$, the quantities $\frac{1}{\epsilon}\|\psi(\boldsymbol{c})-\psi(\boldsymbol{z})\|=\frac{1}{\epsilon}\|\boldsymbol{c}-\boldsymbol{z}\|$ are distributed as the norms of points uniformly drawn from $\boldsymbol{B}_{d}(\mathbf{0}, 1)$. This fact ensures that Equation (1) holds in $\boldsymbol{B}_{d}(\boldsymbol{c}, \epsilon)$ for $r=\frac{1}{\epsilon}\|\boldsymbol{c}-\boldsymbol{z}\|$.

To further generalize our theoretical results, we consider a locally isometric smooth map $\psi: \mathcal{M} \rightarrow \Re^{D}$, and samples drawn from $\mathcal{M} \equiv \Re^{d}$ by means of a non-uniform smooth pdf $f: \mathcal{M} \rightarrow \Re^{+}$. Notice that, being $\psi$ a local isometry, it induces a distance function $\delta_{\psi}(\cdot, \cdot)$ representing the metric on $\psi(\mathcal{M})$. Under these assumptions Equation (1) does not represent the correct pdf of the distances. However, without loss of generality, we consider $\boldsymbol{c}=\mathbf{0}_{d} \in \Re^{d}$ and $\psi(\boldsymbol{c})=\mathbf{0}_{D} \in \Re^{D}$, and we show that any smooth pdf $f$ is locally uniform where the probability is not zero. To this aim, assuming $f\left(\mathbf{0}_{d}\right)>0$ and $\boldsymbol{z} \in \Re^{d}$, we denote with $f_{\epsilon}$ the pdf obtained by setting $f_{\epsilon}(\boldsymbol{z})=0$ when $\|\boldsymbol{z}\|>1$, and $f_{\epsilon}(\boldsymbol{z}) \propto f(\epsilon \boldsymbol{z})$ when $\|\boldsymbol{z}\| \leq 1$. More precisely, denoting with $\chi_{\boldsymbol{B}_{d}(\mathbf{0}, 1)}$ the indicator function on the ball $\boldsymbol{B}_{d}(\mathbf{0}, 1)$, we obtain:

$$
f_{\epsilon}(\boldsymbol{z})=\frac{f(\epsilon \boldsymbol{z}) \chi_{\mathcal{B}_{d}(\mathbf{0}, 1)}(\boldsymbol{z})}{\int_{\boldsymbol{t} \in \mathcal{B}_{d}(\mathbf{0}, 1)} f(\epsilon \boldsymbol{t}) d \boldsymbol{t}}
$$

Theorem 1. Given $\left\{\epsilon_{i}\right\} \rightarrow 0^{+}$, Equation (2) describes a sequence of pdf having the unit d-dimensional ball as support; such sequence converges uniformly to the uniform distribution $\mathbf{B}_{d}$ in the ball $\boldsymbol{B}_{d}(\mathbf{0}, 1)$.

Proof. Evaluating the limit for $\epsilon \rightarrow 0^{+}$of the distance between $f_{\epsilon}$ and $\mathbf{B}_{d}$ in the supremum norm we get:

$$
\begin{aligned}
\lim _{\epsilon \rightarrow 0^{+}}\left\|f_{\epsilon}(\boldsymbol{z})-\mathbf{B}_{d}(\boldsymbol{z})\right\|_{\text {sup }} & =\lim _{\epsilon \rightarrow 0^{+}}\left\|\frac{f(\epsilon \boldsymbol{z}) \chi_{\mathcal{B}_{d}(\mathbf{0}, 1)}}{\int_{\mathcal{B}_{d}(\mathbf{0}, 1)} f(\epsilon \boldsymbol{t}) d \boldsymbol{t}}-\frac{\chi_{\mathcal{B}_{d}(\mathbf{0}, 1)}}{\int_{\mathcal{B}_{d}(\mathbf{0}, 1)} d \boldsymbol{t}}\right\|_{\text {sup }} \\
\{\text { just notation }\} & =\lim _{\epsilon \rightarrow 0^{+}}\left\|\frac{f(\epsilon \boldsymbol{z})}{\int_{\mathcal{B}_{d}(\mathbf{0}, 1)} f(\epsilon \boldsymbol{t}) d \boldsymbol{t}}-\frac{1}{\int_{\mathcal{B}_{d}(\mathbf{0}, 1)} d \boldsymbol{t}}\right\|_{\sup _{\mathcal{B}_{d}(\mathbf{0}, 1)}} \\
\left\{\text { setting } V=\int_{\mathcal{B}_{d}(0,1)} d \boldsymbol{t}\right\} & =\lim _{\epsilon \rightarrow 0^{+}}\left\|\frac{V f(\epsilon \boldsymbol{z})-\int_{\mathcal{B}_{d}(\mathbf{0}, 1)} f(\epsilon \boldsymbol{t}) d \boldsymbol{t}}{V \int_{\mathcal{B}_{d}(\mathbf{0}, 1)} f(\epsilon \boldsymbol{t}) d \boldsymbol{t}}\right\|_{\sup _{\mathcal{B}_{d}(\mathbf{0}, 1)}} \\
\left\{0<\lim _{\epsilon \rightarrow 0^{+}} V \int_{\mathcal{B}_{d}(0,1)} f(\epsilon t) d t<\infty\right\} & =\lim _{\epsilon \rightarrow 0^{+}}\left\|V f(\epsilon \boldsymbol{z})-\int_{\mathcal{B}_{d}(\mathbf{0}, 1)} f(\epsilon \boldsymbol{t}) d \boldsymbol{t}\right\| \|_{\sup _{\mathcal{B}_{d}(\mathbf{0}, 1)}}
\end{aligned}
$$

Defining:

$$
\min (\epsilon)=\min _{\mathcal{B}_{d}(\mathbf{0}, 1)} f(\epsilon \boldsymbol{z}) \quad \max (\epsilon)=\max _{\mathcal{B}_{d}(\mathbf{0}, 1)} f(\epsilon \boldsymbol{z})
$$

and noting that $\min (\epsilon)>0$ definitely since $f\left(\mathbf{0}_{d}\right)>0$, we have:

$$
\begin{aligned}
V \cdot \min (\epsilon) & \leq \quad V f(\epsilon \boldsymbol{z}) \quad \leq V \cdot \max (\epsilon) \\
V \cdot \min (\epsilon) & \leq \int_{\mathcal{B}_{d}(\mathbf{0}, 1)} f(\epsilon \boldsymbol{t}) d \boldsymbol{t} \leq V \cdot \max (\epsilon)
\end{aligned}
$$

thus their difference is bounded by $V(\max (\epsilon)-\min (\epsilon)) \underset{\epsilon \rightarrow 0^{+}}{\longrightarrow} 0^{+}$. 
Theorem1 1 proves that the convergence of $f_{\epsilon}$ to $\mathbf{B}_{d}$ is uniform, and when $\epsilon \rightarrow 0^{+}$ the pdf related to the geodetic distances $\frac{1}{\epsilon} \delta_{\psi}(\psi(\boldsymbol{c}), \psi(\boldsymbol{z}))=\frac{1}{\epsilon}\|\boldsymbol{c}-\boldsymbol{z}\|$ converges to the pdf $g$ reported in Equation (1).

\subsection{Maximum Likelihood Approaches}

Consider a manifold $\mathcal{M} \equiv \Re^{d}$ embedded in a higher dimensional space $\Re^{D}$ through a locally isometric non-linear smooth map $\psi: \mathcal{M} \rightarrow \Re^{D}$. Given a sample set $\boldsymbol{X}_{N}=\left\{\boldsymbol{x}_{i}\right\}_{i=1}^{N}=\left\{\psi\left(\boldsymbol{z}_{i}\right)\right\}_{i=1}^{N} \subset \Re^{D}$, where $\boldsymbol{z}_{i}$ are independent identically distributed points drawn from $\mathcal{M}$ according to a non-uniform smooth pdf $f$ : $\mathcal{M} \rightarrow \Re^{+}$, for each point $\boldsymbol{x}_{i} \in \boldsymbol{X}_{N}$ we find the set of $k+1(1 \leq k \leq N-1)$ nearest neighbors $\overline{\boldsymbol{X}}_{k+1}=\overline{\boldsymbol{X}}_{k+1}\left(\boldsymbol{x}_{i}\right)=\left\{\boldsymbol{x}_{j}\right\}_{j=1}^{k+1} \subset \boldsymbol{X}_{N}$. Calling $\hat{\boldsymbol{x}}=\hat{\boldsymbol{x}}_{k+1}\left(\boldsymbol{x}_{i}\right) \in \overline{\boldsymbol{X}}_{k+1}$ the most distant point from $\boldsymbol{x}_{i}$, we calculate the distance between $\boldsymbol{x}_{i}$ and the nearest neighbor in $\overline{\boldsymbol{X}}_{k+1}$ and we normalize it by means of the distance between $\boldsymbol{x}_{i}$ and $\hat{\boldsymbol{x}}$. More precisely, we have:

$$
\rho\left(\boldsymbol{x}_{i}\right)=\min _{\boldsymbol{x}_{j} \in \overline{\boldsymbol{X}}_{k+1}} \frac{\left\|\boldsymbol{x}_{i}-\boldsymbol{x}_{j}\right\|}{\left\|\boldsymbol{x}_{i}-\hat{\boldsymbol{x}}\right\|}
$$

Theorem 4 in 3 ensures that geodetic distances in the infinitesimal ball converge to Euclidean distances with probability 1; moreover, recalling the result reported in Theorem 1, it is possible to notice that, for $\boldsymbol{x}_{i} \neq \hat{\boldsymbol{x}}$, the quantities $\rho\left(\boldsymbol{x}_{i}\right)$ are samples drawn from the pdf reported in Equation (1), where the parameter $k$ is known and the parameter $d$ must be estimated. A simple approach for the estimation of $d$ is the maximization of the log-likelihood function:

$$
\begin{aligned}
l l(d)= & \sum_{\boldsymbol{x}_{i} \in \boldsymbol{X}_{N}} \log g\left(\boldsymbol{x}_{i} ; k, d\right)=N \log k+N \log d+ \\
& (d-1) \sum_{\boldsymbol{x}_{i} \in \boldsymbol{X}_{N}} \log \rho\left(\boldsymbol{x}_{i}\right)+(k-1) \sum_{\boldsymbol{x}_{i} \in \boldsymbol{X}_{N}} \log \left(1-\rho^{d}\left(\boldsymbol{x}_{i}\right)\right)
\end{aligned}
$$

To select an integer value in $\hat{d} \in\{1 . . D\}$ as the estimated id, it suffices to evaluate $\hat{d}=\arg \max _{d \in\{1 . . D\}} l l(d)$; we call this estimator MIND $_{\mathrm{MLi}}$. On the other side, if a real value is required as a fractal id estimation, the maximal value in $[1, D]$ must be found. To this aim we compute the first derivative of $l l(d)$ and we determine the solutions of $\frac{\partial l l}{\partial d}=0$, thus obtaining:

$$
\frac{N}{d}+\sum_{\boldsymbol{x}_{i} \in \boldsymbol{X}_{N}}\left(\log \rho\left(\boldsymbol{x}_{i}\right)-(k-1) \frac{\rho^{d}\left(\boldsymbol{x}_{i}\right) \log \rho\left(\boldsymbol{x}_{i}\right)}{1-\rho^{d}\left(\boldsymbol{x}_{i}\right)}\right)=0
$$

We recall that the well-known MLE technique adopts a similar derivation since it extracts distance information from all the first $k$ nearest neighbors. We note that, in the particular case $k=1$, the solution of Equation (5) is:

$$
\hat{d}=-\left(\frac{1}{N} \sum_{\boldsymbol{x}_{i} \in \boldsymbol{X}_{N}} \log \rho\left(\boldsymbol{x}_{i}\right)\right)^{-1}
$$


that is exactly the MLE estimator proposed in [18] when $k=1$; we call this estimator MIND $_{\mathrm{ML} 1}$ and its time complexity is $O(D N \log N)$.

For $k>1$ we numerically solve the following optimization problem:

$$
\hat{d}=\underset{1 \leq d \leq D}{\arg \max } l l(d)
$$

To solve this maximization problem we employed the constrained optimization method proposed in 2] with the initial (integer) value $d_{0}=\arg \max _{d \in\{1 . . D\}} l l(d)$. We call this estimator MIND $\mathrm{MLk}_{\text {; }}$; its time complexity is $O\left(D^{2} N \log N\right)$.

\subsection{A pdf Comparison Approach}

In Section 3.2 we presented maximum likelihood estimators for the parameter $d$ (id) in the pdf reported in Equation (11). Notice that, once $k$ is fixed, Equation (11) represents a finite family of $D$ pdfs for all the parameter values $1 \leq d \leq D$. Exploiting this fact, another approach for the estimation of the missing parameter $d$ is the comparison between the $D$ possible theoretical pdfs and a density function estimated by means of the given data.

Consider $\mathcal{M}$ to be a $d$-dimensional hypersphere embedded in the Euclidean space $\Re^{D}$; moreover, denote with $\hat{g}(r ; k)$ an estimation of $g(r ; k, d)$ computed by solely using the sample data points and therefore independent from $d$. The estimation $\hat{d}$ is computed by choosing the dimensionality which minimizes the Kullback-Leibler divergence between $g$ and $\hat{g}$ :

$$
\hat{d}=\underset{1 \leq d \leq D}{\arg \min } \int_{0}^{1} \hat{g}(r ; k) \log \left(\frac{\hat{g}(r ; k)}{g(r ; k, d)}\right) d r
$$

The function $\hat{g}$ can be obtained by means of a set of sample data points as a parametric model; nevertheless, as shown in [6], the number of sample points required to perform dimensionality estimation grows exponentially with the value of the id ("curse of dimensionality"). For this reason, when the dimensionality is too high, the number of sample points practically available is insufficient to compute an acceptable estimation. Moreover, the fraction between the points on (or close to) the edge of the manifold, and the other points (inside the manifold) increases in probability when the dimensionality increases ("edge effect", see [24]), thus affecting the results achieved by estimators based on statistics related to the behavior of point neighborhoods, such as the algorithms proposed in Section 3.2 and MLE.

To address these problems in literature few approaches have been proposed, among which we recall [1]. In this work, Camastra et al. propose a correction of the estimated id based on the estimation of the error obtained on synthetically produced datasets of known dimensionality (hypercubes).

In our work, to reduce the bias between the analytical pdf $g$ and the estimated one $\hat{g}$, for each value $1 \leq d \leq D$ we learn a test pdf $\check{g}_{d}(r ; k)$ by means of points uniformly drawn from the $d$-dimensional unit hypersphere; moreover, to best resemble the point density of the given dataset, we draw exactly $N$ points per 
dimensionality. Finally, we numerically estimate the Kullback-Leibler divergence by means of the estimates $\hat{g}$ and $\check{g}_{d}$.

More precisely, consider a manifold $\mathcal{M} \equiv \Re^{d}$ embedded in a higher dimensional space $\Re^{D}$ through a locally isometric non-linear smooth map $\psi: \mathcal{M} \rightarrow$ $\Re^{D}$. Given a sample set $\boldsymbol{X}_{N}=\left\{\boldsymbol{x}_{i}\right\}_{i=1}^{N}=\left\{\psi\left(\boldsymbol{z}_{i}\right)\right\}_{i=1}^{N} \subset \Re^{D}$ where $\boldsymbol{z}_{i}$ are independent identically distributed points drawn from $\mathcal{M}$ according to a nonuniform smooth pdf $f: \mathcal{M} \rightarrow \Re^{+}$, we compute a vector of normalized distances $\hat{\boldsymbol{r}}=\left\{\hat{r}_{i}\right\}_{i=1}^{N}=\left\{\rho\left(\boldsymbol{x}_{i}\right)\right\}_{i=1}^{N}$ by means of Equation (3). Moreover, for each dimensionality $d \in\{1 . . D\}$ we uniformly draw a set of $N$ points $\boldsymbol{Y}_{N d}=\left\{\boldsymbol{y}_{i}\right\}_{i=1}^{N}$ from the unit $d$-dimensional hypersphere, and we similarly compute a vector of normalized distances $\check{\boldsymbol{r}}_{d}=\left\{\check{r}_{i d}\right\}_{i=1}^{N}=\left\{\rho\left(\boldsymbol{y}_{i}\right)\right\}_{i=1}^{N}$. Notice that, a $d$-dimensional vector randomly sampled from a $d$ dimensional hypersphere according to the uniform pdf, can be generated by drawing a point $\overline{\boldsymbol{y}}$ from a standard normal distribution $\mathcal{N}\left(\cdot \mid \mathbf{0}_{d}, 1\right)$ and by scaling its norm (see Section 3.29 of [9]):

$$
\boldsymbol{y}=\frac{u^{\frac{1}{d}}}{\|\overline{\boldsymbol{y}}\|} \overline{\boldsymbol{y}}, \quad \overline{\boldsymbol{y}} \sim \mathcal{N}\left(\cdot \mid \mathbf{0}_{d}, 1\right)
$$

where $u$ is a random sample drawn from the uniform distribution $U(0,1)$.

Given a set of values $r_{i=1}^{N} \subset[0,1]$ distributed according to the pdf $p$, in [25] the following pdf estimator is proposed:

$$
\hat{p}(r)=\frac{N^{-1}}{2 \rho(r)}
$$

where $\rho(r)$ is the distance between $r$ and its nearest neighbor. In our problem, considering a distance $\hat{r}_{i} \in \hat{\boldsymbol{r}}$, the pdf estimates $\hat{g}$ and $\check{g}_{d}$ can be computed as follows:

$$
\hat{g}\left(\hat{r}_{i} ; k\right)=\frac{1 /(N-1)}{2 \hat{\rho}\left(\hat{r}_{i}\right)} \quad \check{g}_{d}\left(\hat{r}_{i} ; k\right)=\frac{1 / N}{2 \check{\rho}_{d}\left(\hat{r}_{i}\right)}
$$

where $\hat{\rho}\left(\hat{r}_{i}\right)$ and $\check{\rho}_{d}\left(\hat{r}_{i}\right)$ are the distances between $\hat{r}_{i}$ and its first neighbor in $\hat{\boldsymbol{r}}$ and in $\check{\boldsymbol{r}}_{d}$ respectively.

In 25] a Kullback-Leibler divergence estimator based on the nearest neighbor search is proposed; moreover, the authors show that their method is more effective than partitioning-based techniques, especially when the number of samples is limited. Employing this estimator between $\hat{g}$ and $\check{g}_{d}$ we obtain:

$$
\begin{aligned}
\hat{K} L\left(\hat{g}, \check{g}_{d}\right) & =\frac{1}{N} \sum_{i=1}^{N} \log \frac{\hat{g}\left(\hat{r}_{i} ; k\right)}{\check{g}_{d}\left(\hat{r}_{i} ; k\right)}=\frac{1}{N} \sum_{i=1}^{N} \log \frac{\frac{1 /(N-1)}{2 \hat{\rho}\left(\hat{r}_{i}\right)}}{\frac{1 / N}{2 \check{\rho}_{d}\left(\hat{r}_{i}\right)}} \\
& =\log \frac{N}{N-1}+\frac{1}{N} \sum_{i=1}^{N} \log \frac{\hat{\rho}\left(\hat{r}_{i}\right)}{\check{\rho}_{d}\left(\hat{r}_{i}\right)}
\end{aligned}
$$

Employing Equation (12), the estimated id value $(\hat{d})$ is computed as follows:

$$
\hat{d}=\underset{d \in\{1 . . D\}}{\arg \min }\left(\log \frac{N}{N-1}+\frac{1}{N} \sum_{i=1}^{N} \log \frac{\hat{\rho}\left(\hat{r}_{i}\right)}{\check{\rho}_{d}\left(\hat{r}_{i}\right)}\right)
$$


Table 1. Brief description of the 15 synthetic and 3 real datasets, where $d$ is the id and $D$ is the embedding space dimension. In the synthetic datasets' name, the number in the subscript refers to the dataset name used by the generator proposed in [13.

\begin{tabular}{|c|c|c|c|l|}
\hline Dataset & Name & $\boldsymbol{d}$ & $\boldsymbol{D}$ & Description \\
\hline \hline \multirow{5}{*}{ Syntethic } & $\mathcal{M}_{1}$ & 10 & 11 & Uniformly sampled sphere linearly embedded. \\
& $\mathcal{M}_{2}$ & 3 & 5 & Affine space. \\
& $\mathcal{M}_{3}$ & 4 & 6 & Concentrated figure, confusable with a 3d one. \\
& $\mathcal{M}_{4}$ & 4 & 8 & Non-linear manifold. \\
& $\mathcal{M}_{5}$ & 2 & 3 & 2-d Helix \\
& $\mathcal{M}_{6}$ & 6 & 36 & Non-linear manifold. \\
& $\mathcal{M}_{7}$ & 2 & 3 & Swiss-Roll. \\
& $\mathcal{M}_{9}$ & 12 & 72 & Non-linear manifold. \\
& $\mathcal{M}_{10 a}$ & 10 & 20 & Affine space. \\
& $\mathcal{M}_{10 b}$ & 17 & 18 & Uniformly sampled hypercube. \\
& $\mathcal{M}_{10 c}$ & 24 & 25 & Uniformly sampled hypercube. \\
& $\mathcal{M}_{11}$ & 2 & 3 & Möebius band 10-times twisted. \\
& $\mathcal{M}_{12}$ & 20 & 20 & Isotropic multivariate Gaussian. \\
& $\mathcal{M}_{13}$ & 1 & 13 & Curve. \\
\hline \hline \multirow{5}{*}{ Real } & $\mathcal{M}_{\text {Faces }}$ & 3 & 4096 & ISOMAP face dataset. \\
& $\mathcal{M}_{\text {MNIST1 }}$ & $8-11$ & 784 & MnIST database (digit 1). \\
& $\mathcal{M}_{\text {Santafe }}$ & 9 & 50 & Santa Fe dataset (version D2). \\
\hline
\end{tabular}

We call this estimator MIND $\mathrm{KL}$; its time complexity is $O\left(D^{2} N \log N\right)$. To obtain a stable id estimation we execute $\operatorname{MiND}_{\mathrm{KL}} 20$ times for each dataset and we average the obtained results.

Due to Theorem 1, Theorem 4 in [3], and considering that the Kullback-Leibler divergence estimator employed is consistent as shown in [25], Equation (13) represents a consistent estimator for the intrinsic dimensionality of the manifold $\mathcal{M}$.

\section{Algorithm Evaluation}

In this section we describe the datasets employed in our experiments (see Section 4.1), we summarize the adopted experimental settings (see Section 4.2), and we report the achieved results (see Section 4.3).

\subsection{Dataset Description}

To evaluate our algorithms, we have performed experiments on both 15 synthetic and 3 real datasets (see Table 11). To generate the synthetic datasets we have employed the tool proposed in [13. Instead, the real datasets are the ISOMAP face database [23, the MNIST database [16] and the Santa Fe 21] dataset.

The ISOMAP face database consists in 698 gray-level images of size $64 \times 64$ depicting the face of a sculpture. This dataset has three degrees of freedom: two for the pose and one for the lighting direction. 
The MNIST database consists in 70000 gray-level images of size $28 \times 28$ of hand-written digits; in our tests we used the 6742 training points representing the digit 1 . The id of this database is not actually known, but some works have proposed similar estimations [133] for the different digits; considering digit 1, the proposed id values are in the range $\{8 . .11\}$.

The version $D 2$ of the Santa Fe dataset is a synthetic time series of 50000 one-dimensional points; it was generated by a simulation of particle motion, and it has nine degrees of freedom. In order to estimate the attractor dimension of this time series, we used the method of delays described in [7], which generates $D$ dimensional vectors by collecting $D$ values from the original dataset; by choosing $D=50$ we obtained a dataset containing 1000 points in $\Re^{50}$.

\subsection{Experimental Setting}

We compared our method with well-known id estimators: PCA, kNNG, CD, MLE, and Hein. For kNNG, MLE, and Hein 1 we used the authors' Matlab implementation, whilst for the other algorithms we employed the version provided by the dimensionality reduction toolbox 2 .

To generate the synthetic datasets we adopted the generator described in [13. creating 20 instances of each dataset reported in Table 1, each of which is composed by 2000 randomly sampled points. For each technique, to obtain a stable estimation, we averaged the results achieved on the different subsets. To execute multiple tests on $\mathcal{M}_{\text {MnIST1 }}$, we extracted from the digit 1 dataset 5 random subsets containing 2000 points each.

Table 2. Parameter settings for the different estimators: $k$ represents the number of neighbors, $\gamma$ the edge weighting factor for kNN, $M$ the number of Least Square (LS) runs, and $N$ the number of resampling trials per LS iterations

\begin{tabular}{|c|c|c|}
\hline Method & Synthetic & Real \\
\hline \hline PCA & Threshold $=0.025$ & Threshold $=0.0025$ \\
CD & None & None \\
MLE & $k_{1}=6 k_{2}=20$ & $k_{1}=3 k_{2}=8$ \\
$\mathrm{kNNG}_{1}$ & $k_{1}=6, k_{2}=20, \gamma=1, M=1, N=10$ & $k_{1}=3, k_{2}=8, \gamma=1, M=1, N=10$ \\
$\mathrm{kNNG}_{2}$ & $k_{1}=6, k_{2}=20, \gamma=1, M=10, N=1$ & $k_{1}=3, k_{2}=8, \gamma=1, M=10, N=1$ \\
MiND $_{\mathrm{ML} 1}$ & $k=1$ & $k=1$ \\
MiND $_{\mathrm{MLk}}$ & $k=10$ & $k=5$ \\
MiND $_{\mathrm{MLi}}$ & $k=10$ & $k=5$ \\
MiND $_{\mathrm{KL}}$ & $k=10$ & $k=5$ \\
\hline
\end{tabular}

In Table 2 the employed configuration parameters are summarized. To relax the dependency of kNNG from the selection of its parameter $k$, we performed

\footnotetext{
${ }^{1}$ http://www.eecs.umich.edu/ hero/IntrinsicDim/, http://www.stat.lsa.umich.edu/ elevina/mledim.m, http://www.ml.uni-saarland.de/code.shtml

${ }^{2}$ http://cseweb.ucsd.edu/ lvdmaaten/dr/download.php
} 
multiple runs with $k_{1} \leq k \leq k_{2}$ (see Table 2) and we averaged the achieved results.

\subsection{Experimental Results}

In this section the results achieved on both real and synthetic datasets are reported.

In Table 3 the results achieved on the synthetic datasets are summarized. As can be noticed, all the algorithms but PCA achieve good results for datasets with low dimensionality $(d<10)$, whilst the PCA method obtains bad estimations when dealing with non-linear manifolds, producing overestimates. With high dimensional datasets, all the techniques but PCA and $M i N D_{K L}$ achieve bad results generally underestimating the id due to the edge-effect and the insufficient cardinality of the datasets. Notice that, $\mathrm{MiND}_{\mathrm{KL}}$ achieves good approximations both on low and high intrinsic dimensional datasets, dealing with both linear and non-linear embeddings, thus obtaining results always comparable with those that better approximate the id. In the last row of Table 3 the Mean Percentage Error (MPE) indicator is reported; for each algorithm this value is computed as the mean of the percentage errors obtained on each dataset:

$$
\text { MPE }=\frac{100}{\# \mathcal{M}} \sum_{\mathcal{M}} \frac{\left|\hat{d}_{\mathcal{M}}-d_{\mathcal{M}}\right|}{d_{\mathcal{M}}}
$$

where $\# \boldsymbol{M}=15$ is the number of tested manifolds (see Table 1). Notice that $\mathrm{MiND}_{\mathrm{KL}}$ obtains the minimum MPE.

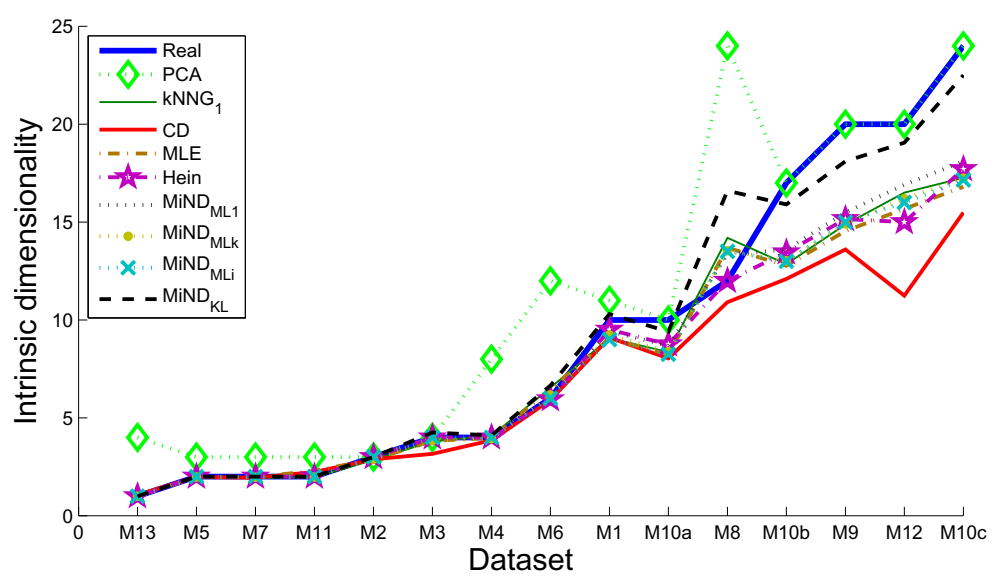

Fig. 1. Comparison between the results achieved by the id estimators with respect to the increase of the manifolds' id 
Table 3. Results achieved on the synthetic datasets. The last row contains the MPE indicator that allows to perform a direct comparison among methods. The best approximations are highlighted in bold case.

\begin{tabular}{|c|c||c|c|c|c|c|c|c|c|c|c|}
\hline Dataset & $d$ & PCA & kNNG $_{1}$ & kNNG $_{2}$ & CD & MLE & Hein & MiND $_{\text {ML } 1}$ & MiND & & MiND \\
MLi & MiND \\
KL \\
\hline \hline $\mathcal{M}_{13}$ & 1 & 4.00 & $\mathbf{1 . 0 0}$ & 1.01 & 1.07 & $\mathbf{1 . 0 0}$ & $\mathbf{1 . 0 0}$ & $\mathbf{1 . 0 0}$ & $\mathbf{1 . 0 0}$ & $\mathbf{1 . 0 0}$ & $\mathbf{1 . 0 0}$ \\
$\mathcal{M}_{5}$ & 2 & 3.00 & 1.96 & $\mathbf{2 . 0 0}$ & 1.98 & 1.96 & $\mathbf{2 . 0 0}$ & 1.97 & 1.97 & $\mathbf{2 . 0 0}$ & $\mathbf{2 . 0 0}$ \\
$\mathcal{M}_{7}$ & 2 & 3.00 & 1.93 & 1.98 & 1.94 & 1.97 & $\mathbf{2 . 0 0}$ & 1.98 & 1.96 & $\mathbf{2 . 0 0}$ & $\mathbf{2 . 0 0}$ \\
$\mathcal{M}_{11}$ & 2 & 3.00 & 1.96 & 2.01 & 2.23 & 2.30 & $\mathbf{2 . 0 0}$ & 1.97 & 1.97 & $\mathbf{2 . 0 0}$ & $\mathbf{2 . 0 0}$ \\
$\mathcal{M}_{2}$ & 3 & $\mathbf{3 . 0 0}$ & 2.85 & 2.93 & 2.88 & 2.87 & $\mathbf{3 . 0 0}$ & 2.93 & 2.88 & $\mathbf{3 . 0 0}$ & $\mathbf{3 . 0 0}$ \\
$\mathcal{M}_{3}$ & 4 & $\mathbf{4 . 0 0}$ & 3.80 & 4.22 & 3.16 & 3.82 & $\mathbf{4 . 0 0}$ & 3.89 & 3.84 & $\mathbf{4 . 0 0}$ & 4.25 \\
$\mathcal{M}_{4}$ & 4 & 8.00 & 4.08 & 4.06 & 3.85 & 3.98 & $\mathbf{4 . 0 0}$ & 3.95 & 3.93 & $\mathbf{4 . 0 0}$ & 4.10 \\
$\mathcal{M}_{6}$ & 6 & 12.00 & 6.53 & 13.99 & 5.91 & 6.45 & 5.95 & 5.91 & 6.17 & $\mathbf{6 . 0 0}$ & 6.65 \\
$\mathcal{M}_{1}$ & 10 & 11.00 & 9.07 & 9.39 & 9.09 & 9.06 & 9.50 & 9.41 & 9.23 & 9.00 & $\mathbf{1 0 . 3 0}$ \\
$\mathcal{M}_{10 a}$ & 10 & $\mathbf{1 0 . 0 0}$ & 8.35 & 9.00 & 8.04 & 8.22 & 8.75 & 8.68 & 8.38 & 8.25 & 9.40 \\
$\mathcal{M}_{8}$ & 12 & 24.00 & 14.19 & 8.29 & 10.91 & 13.69 & $\mathbf{1 2 . 0 0}$ & 13.35 & 13.53 & 13.50 & 16.60 \\
$\mathcal{M}_{10 b}$ & 17 & $\mathbf{1 7 . 0 0}$ & 12.85 & 15.58 & 12.09 & 12.77 & 13.45 & 13.59 & 13.02 & 13.00 & 15.90 \\
$\mathcal{M}_{9}$ & 20 & $\mathbf{2 0 . 0 0}$ & 14.87 & 17.07 & 13.60 & 14.54 & 15.15 & 15.49 & 14.90 & 15.00 & 18.10 \\
$\mathcal{M}_{12}$ & 20 & $\mathbf{2 0 . 0 0}$ & 16.50 & 14.58 & 11.24 & 15.67 & 15.00 & 16.91 & 16.19 & 16.00 & 19.05 \\
$\mathcal{M}_{10 c}$ & 24 & $\mathbf{2 4 . 0 0}$ & 17.26 & 23.68 & 15.48 & 16.80 & 17.70 & 18.10 & 17.24 & 17.15 & 22.50 \\
\hline \hline MPE & & 50.67 & 11.20 & 16.23 & 15.38 & 12.03 & 7.65 & 8.32 & 10.02 & 9.14 & $\mathbf{6 . 2 6}$ \\
\hline
\end{tabular}

In Table 4 the results achieved by the tested algorithms is reported 3 with respect to the increase of the manifolds' id. This figure confirms that the PCA estimator cannot correctly deal with non-linear manifolds producing strong estimation errors (overestimates). Moreover, this figure underlines that most of the other techniques are able to produce precise estimations when the id is low, whilst they strongly underestimate when the id is sufficiently high. The only exception is given by the results of $\mathrm{MiND}_{\mathrm{KL}}$; indeed, this method allows to obtain stable estimations both on low and high id datasets.

Table 4. Results achieved on the real datasets by the employed approaches. The best approximations are highlighted in bold case.

\begin{tabular}{|c|c||c|c|c|c|c|c|c|c|c|c|}
\hline Dataset & $d$ & PCA & kNNG $_{1}$ & kNNG $_{2}$ & CD & MLE & Hein & MiND $_{\text {ML1 }}$ & MiND $_{\text {MLk }}$ & MiND $_{\text {MLi }}$ & MiND $_{\text {KL }}$ \\
\hline \hline $\mathcal{M}_{\text {Faces }}$ & 3 & 21.00 & 3.60 & 4.32 & 3.37 & 4.05 & $\mathbf{3 . 0 0}$ & 3.52 & 3.59 & 4.00 & 3.90 \\
$\mathcal{M}_{\text {MNIST1 }}$ & $8-11$ & 11.80 & 10.37 & 9.58 & 6.96 & 10.29 & 8.00 & 11.33 & 10.02 & $\mathbf{9 . 4 5}$ & 11.00 \\
$\mathcal{M}_{\text {Santa Fe }}$ & 9 & 18.00 & 7.28 & 7.43 & 4.39 & 7.16 & 6.00 & 6.31 & 6.78 & 7.00 & $\mathbf{7 . 6 0}$ \\
\hline
\end{tabular}

In Table 4 the results achieved on real datasets have been summarized. Notice that also in the case of noisy real datasets, our methods have obtained either the best approximation of the id, or results always comparable with those achieved by the best performing technique.

\footnotetext{
${ }^{3}$ The results of $\mathrm{kNNG}_{2}$ are omitted for clarity; notice that, this technique has obtained worst results with respect to $\mathrm{kNNG}_{1}$.
} 

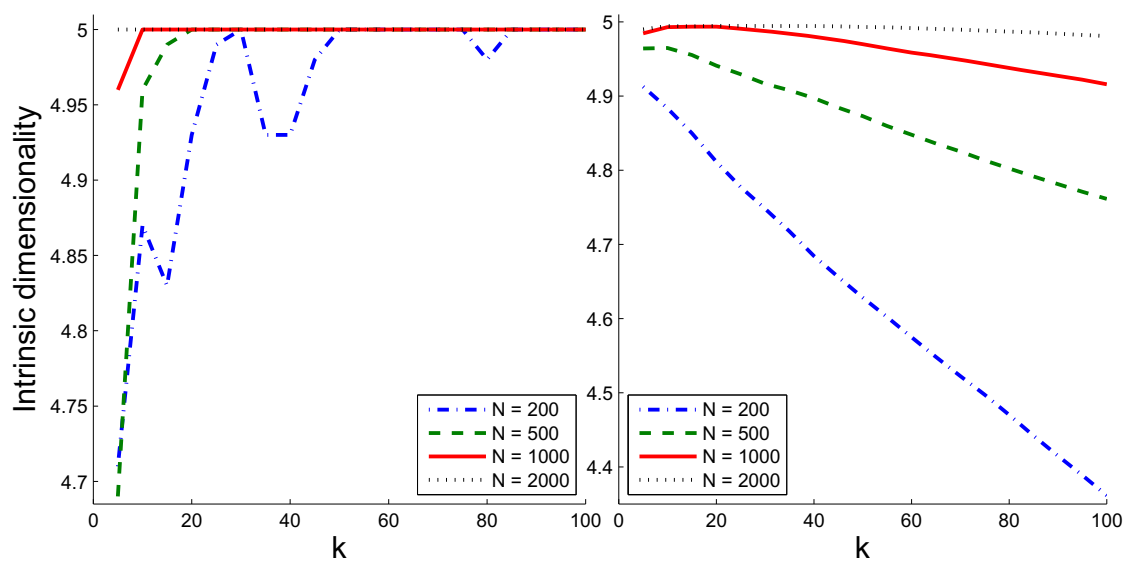

Fig. 2. Behavior of $M N_{\mathrm{KL}}$ (left) and MiND $D_{\mathrm{MLk}}$ (right) applied to points drawn from a 5 -dimensional standard normal distribution, in this test $N \in\{200,500,1000,2000\}$ and $k \in\{5 . .100\}$

Finally, to test the robustness of our algorithms with respect to the choice of the parameter $k$, we reproduced the experiments proposed for the MLE algorithm in Figure 1 (a) of [17] employing $\mathrm{MiND}_{\mathrm{KL}}$ and $\mathrm{MiND}_{\mathrm{MLk}}$, and we averaged the curves obtained by 10 runs. In these tests the adopted datasets are composed by points drawn from the standard Gaussian pdf in $\Re^{5}$. We repeated the test for datasets with cardinalities $N \in\{200,500,1000,2000\}$, and varying the parameter $k$ in the range $\{5 . .100\}$. As shown in 2, $\mathrm{MiND}_{\mathrm{KL}}$ (left) demonstrates to be robust to the choice of its parameter $k$, whilst MiND $_{\mathrm{MLk}}$ (right) shows a behavior comparable to that of MLE (see Figure 1 (a) in [17]).

Concluding, the results achieved on both real and synthetic datasets have confirmed the quality of the proposed methods; more specifically, MiND $\mathrm{KL}_{\mathrm{KL}}$ has proved to be the best estimator since it is robust to the choice of its parameter, it obtains the smallest MPE (see Table 3), and it achieves good approximations both on high and low id datasets.

\section{Conclusions and Future Works}

In this work we proposed Minimum Neighbor Distance estimators of intrinsic dimension. These methods, for each point in the dataset, exploit the pdf related to the normalized distance of its nearest neighbor. More precisely, the algorithms $\mathrm{MiND}_{\mathrm{ML} *}$ are based on the maximization of the log-likelihood function associated to the normalized pdf of the distances, whilst the estimator MiND $\mathrm{KL}_{\mathrm{KL}}$ compares the estimated pdf with those of random points uniformly drawn from unitary hyperspheres with dimensionality in $\{1 . . D\}$.

We tested our algorithms on synthetic and real datasets comparing them with state of the art id estimators. The achieved results demonstrate that our 
techniques are promising, and the Mean Percentage Error indicator underlines the stability of the proposed methods. We note that, among the techniques we described, our experiments demonstrate that $\mathrm{MiND}_{\mathrm{KL}}$ is the most robust estimator, since it deals with both low and high id, and it manages both linear and non-linear manifolds, achieving results always comparable to the best estimations. Furthermore, its performances are not strongly affected by the choice of its only parameter $k$, and it shows to be resistant to the curse of dimensionality.

In Equation (3) the kNN Euclidean distances are normalized by means of the maximum one. In the limit this normalization factor converges to the geodetic one, but for a finite set of sample points an approximation is introduced. In future works we want to investigate the behavior of this approximation to reduce its negative effect on the id estimation.

\section{References}

1. Camastra, F., Vinciarelli, A.: Estimating the intrinsic dimension of data with a fractal-based method. IEEE Trans. Pattern Anal. Mach. Intell. 24, 1404-1407 (2002)

2. Coleman, T.F., Li, Y.: An interior, trust region approach for nonlinear minimization subject to bounds. SIAM Journal on Optimization 6, 418-445 (1996)

3. Costa, J., Hero, A.O.: Learning intrinsic dimension and entropy of shapes. In: Krim, H., Yezzi, T. (eds.) Statistics and analysis of shapes. Birkhäuser, Basel (2005)

4. Costa, J.A., Hero, A.O.: Geodesic entropic graphs for dimension and entropy estimation in manifold learning. IEEE Transaction on Signal Processing 52(8), 2210 $2221(2004)$

5. Costa, J.A., Hero, A.O.: Learning intrinsic dimension and entropy of highdimensional shape spaces. In: EUSIPCO (2004)

6. Eckmann, J.-P., Ruelle, D.: Fundamental limitations for estimating dimensions and lyapunov exponents in dynamical systems. Physica D: Nonlinear Phenomena 56(23), 185-187 (1992)

7. Edward, O.: Chaos in Dynamical Systems. Cambridge University Press, Cambridge (1993)

8. Farahmand, A.M., Szepesvari, C., Audibert, J.Y.: Manifold-adaptive dimension estimation. In: Proc. of ICML (2007)

9. Fishman, G.S.: Monte Carlo: Concepts, Algorithms, and Applications. Springer Series in Operations Research. Springer, New York (1996)

10. Fukunaga, K.: An algorithm for finding intrinsic dimensionality of data. IEEE Trans. on Computers 20, 176-183 (1971)

11. Fukunaga, K.: Intrinsic Dimensionality Extraction. In: Krishnaiah, P.R., Kanal, L.N. (eds.) Classification, Pattern Recognition and Reduction of Dimensionality. North-Holland, Amsterdam (1982)

12. Grassberger, P., Procaccia, I.: Measuring the strangeness of strange attractors. Physica D: Nonlinear Phenomena 9, 189-208 (1983)

13. Hein, M.: Intrinsic dimensionality estimation of submanifolds in euclidean space. In: ICML, pp. 289-296 (2005)

14. Jollife, I.T.: Principal Component Analysis. Springer Series in Statistics. Springer, New York (1986)

15. Kégl, B.: Intrinsic dimension estimation using packing numbers. In: Becker, S., Thrun, S., Obermayer, K. (eds.) NIPS, pp. 681-688. MIT Press, Cambridge (2002) 
16. LeCun, Y., Bottou, L., Bengio, Y., Haffner, P.: Gradient-Based Learning Applied to Document Recognition. Proceedings of the IEEE 86(11), 2278-2324 (1998)

17. Levina, E., Bickel, P.J.: Maximum likelihood estimation of intrinsic dimension. Ann. Arbor. MI 1, 777-784 (2005)

18. MacKay, D.J.C., Ghahramani, Z.: Comments on 'maximum likelihood estimation of intrinsic dimension' by E. Levina and P. Bickel (2005), http://www.inference.phy.cam.ac.uk/mackay/dimension/

19. Mordohai, P., Medioni, G.: Dimensionality estimation, manifold learning and function approximation using tensor voting. J. Mach. Learn. Res. 11, 411-450 (2010)

20. Pettis, K., Bailey, T., Jain, A., Dubes, R.: An intrinsic dimensionality estimator from near-neighbor information (1979)

21. Pineda, F.J., Sommerer, J.C.: Estimating generalized dimensions and choosing time delays: A fast algorithm. In: Forecasting the Future and Understanding the Past. Time Series Prediction, pp. 367-385 (1994)

22. Roweis, S.T., Saul, L.K.: Nonlinear Dimensionality Reduction by Locally Linear Embedding. Science 290, 2323-2326 (2000)

23. Tenenbaum, J.B., Silva, V., Langford, J.C.: A Global Geometric Framework for Nonlinear Dimensionality Reduction. Science 290, 2319-2323 (2000)

24. Verveer, P.J., Duin, R.P.W.: An evaluation of intrinsic dimensionality estimators. IEEE Transactions on Pattern Analysis and Machine Intelligence 17, 81-86 (1995)

25. Wang, Q., Kulkarni, S.R., Verd, S.: A nearest-neighbor approach to estimating divergence between continuous random vector. In: IEEE Int. Symp. Information Theory (ISIT 2006), pp. 242-246 (2006)

\section{Appendix A Algorithms Implementation}

In this appendix the pseudocode of our algorithms is reported. In Algorithm $1 \mathrm{MiND}_{\mathrm{MLi}}$ is shown, where $k N N\left(\boldsymbol{X}_{N}, \boldsymbol{x}, k\right)$ is the procedure that employs a knearest neighbor search returning the set of the $k$ nearest neighbors of $\boldsymbol{x}$ in $\boldsymbol{X}_{N}$. Since MiND ML1 $_{1}$ consists only in Equation (6), whilst MiND MLk $_{\text {(6) }}$ consists in refining the MiND $D_{\text {MLi }}$ result by means of a constrained optimization algorithm (see Equation (7D), and due to the lack of space, these two algorithms are not reported. Algorithm 2 reports the pseudocode of $\mathrm{MiND}_{\mathrm{KL}}$, where $N N\left(\boldsymbol{X}_{N}, \boldsymbol{x}\right)$ is the procedure that returns the nearest neighbor of $\boldsymbol{x}$ in $\boldsymbol{X}_{N}$. 
Algorithm 1. Pseudocode for the MiND $\mathrm{MLi}_{\text {algorithm. }}$

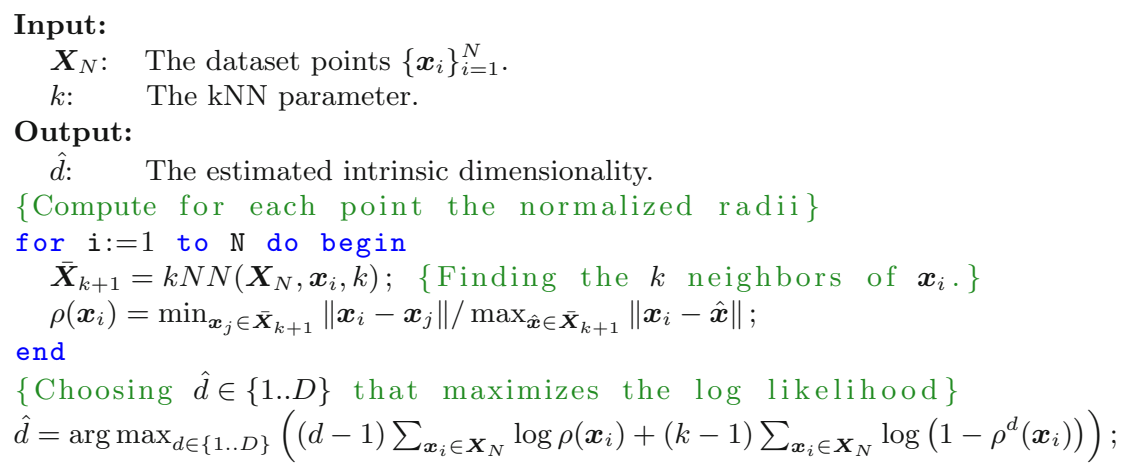

Algorithm 2. Pseudocode for the MiND $\mathrm{KL}_{\mathrm{L}}$ algorithm.

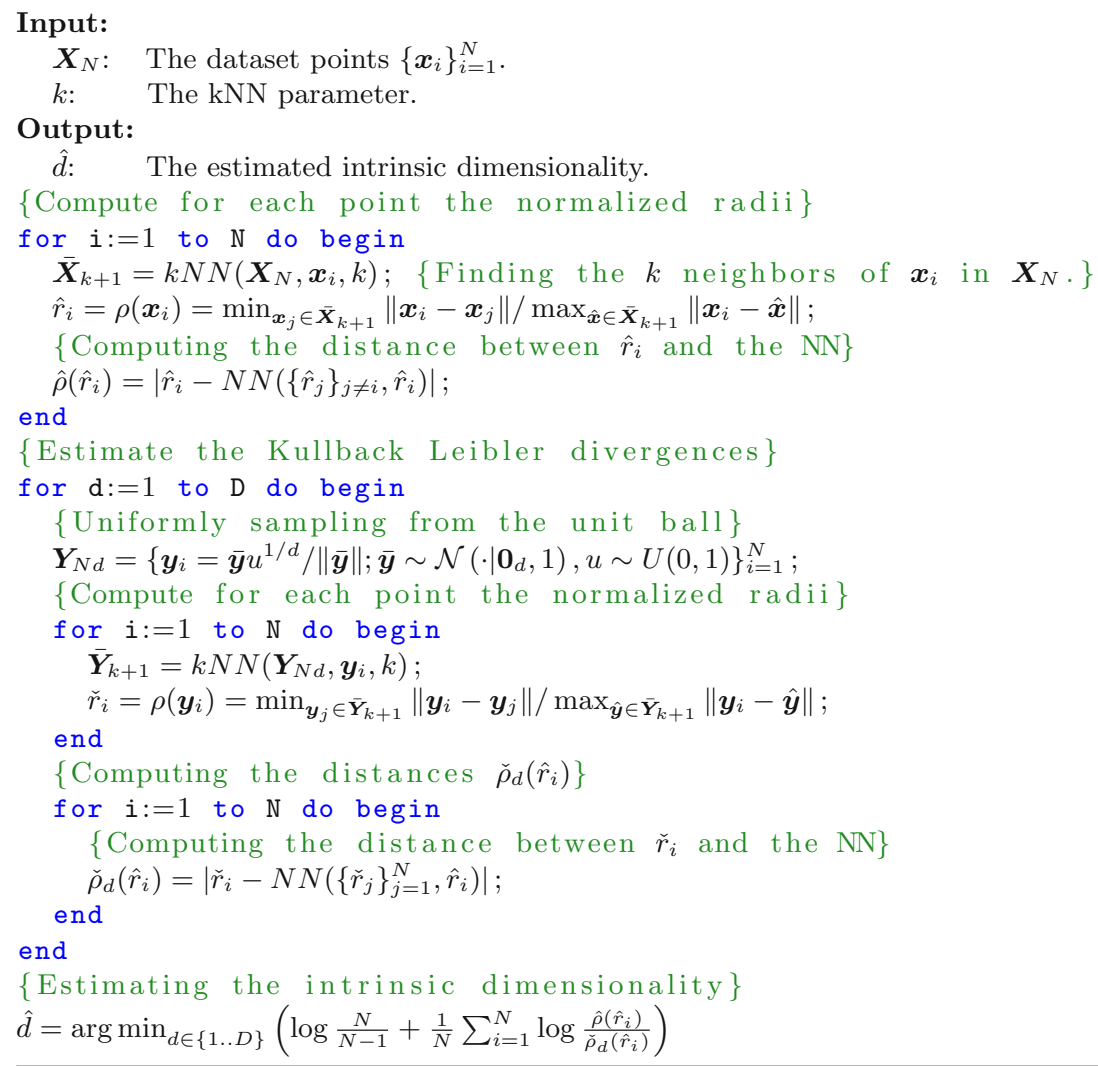

